\title{
Aprendizaje-servicio en la Pontificia Universidad Javeriana seccional Bogotá, una experiencia de institucionalización en curso
}

\author{
A. Barbosa \\ D. García \\ Pontificia Universidad Javeriana, Bogotá, Colombia
}

\section{Resumen}

En la misión y el proyecto educativo de la Pontificia Universidad Javeriana se declara la construcción de una sociedad más justa, sostenible, incluyente y respetuosa de la dignidad humana a través de la formación integral y la producción de conocimiento pertinente. Aunque a lo largo de la historia de la Universidad han existido diferentes experiencias educativas cercanas al aprendizaje-servicio, este no se ha institucionalizado formalmente. En el año 2019 se empezó a considerar esta posibilidad y posteriormente, en el 2020, el Rector suscribió el acuerdo de colaboración interinstitucional con UNISERVITATE y le encargó el liderazgo y gestión del proyecto a la Oficina para el Fomento de la Responsabilidad Social Universitaria. Este artículo tiene por objetivo sistematizar la experiencia para construir una ruta de institucionalización del aprendizaje-servicio. A través de entrevistas semiestructuradas, grupos focales, análisis documental y la técnica de la sistematización, se recogió la información con relación a los sentidos y prácticas asociados al proceso de institucionalización. Ello permitió hacer una triangulación para extraer las necesidades, potencialidades y retos que implica institucionalizar el aprendizajeservicio en la universidad. Se plantea la necesidad de que el aprendizaje servicio tenga un respaldo institucional claro y normalizado.

\section{Palabras clave}

Aprendizaje-servicio, institucionalización, sistematización de experiencias, significados.

Fecha de recepción: 24/XI/2021

Fecha de aceptación: 9/XII/2021 


\title{
Service-learning at the sectional Pontificia Universidad Javeriana Bogotá, an ongoing institutionalization experience
}

\begin{abstract}
The mission and educational project of the Pontificia Universidad Javeriana declares the construction of a more just, sustainable, inclusive and respectful society of human dignity through comprehensive training and the production of relevant knowledge. Although throughout the history of the University there have been different educational experiences close to service-learning, it has not been formally institutionalized. In 2019 this possibility began to be considered and later, in 2020, the Rector signed the inter-institutional collaboration agreement with UNISERVITATE and entrusted the leadership and management of the project to the Office for the Promotion of University Social Responsibility. This article aims to systematize the experience to build a path of institutionalization of service-learning. Through semistructured interviews, focus groups, documentary analysis and the systematization technique, information was collected in relation to the meanings and practices associated with the institutionalization process. This allowed a triangulation to be carried out to extract the needs, potentials and challenges involved in institutionalizing service-learning in the university. There is a need for service-learning to have clear and standardized institutional support.
\end{abstract}

\section{Key words}

Service-learning, institutionalization, systematization of experiences, meanings. 


\section{Aprenentatge servei a la Pontifícia Universidad Javeriana seccional Bogotá, una experiència d'institucionalització en curs}

\section{Resum}

A la missió i el projecte educatiu de la Pontifícia Universidad Javeriana es declara la construcció d'una societat més justa, sostenible, inclusiva i respectuosa de la dignitat humana a través de la formació integral i la producció de coneixement pertinent. Tot i que al llarg de la història de la universitat hi ha hagut diferents experiències educatives properes a l'aprenentatge servei, aquest no s'ha institucionalitzat formalment. L'any 2019 es va començar a considerar aquesta possibilitat i posteriorment, el 2020, el Rector va subscriure l'acord de col-laboració interinstitucional amb UNISERVITATE i li va encarregar el lideratge i la gestió del projecte a l'Oficina per al Foment de la Responsabilitat Social Universitària. Aquest article té com a objectiu sistematitzar l'experiència per construir una ruta d'institucionalització de l'aprenentatge servei. A través d'entrevistes semiestructurades, grups focals, anàlisi documental i la tècnica de la sistematització, es va recollir la informació amb relació als sentits i les pràctiques associades al procés d'institucionalització. Això va permetre fer una triangulació per extreure'n les necessitats, potencialitats i reptes que implica institucionalitzar l'aprenentatge servei a la universitat. Es planteja la necessitat que l'aprenentatge servei tingui un suport institucional clar i normalitzat.

\section{Paraules clau}

Aprenentatge servei, institucionalització, sistematització d’experiències, significats. 


\section{Introducción}

Aunque en los años 60 y 70 la práctica del aprendizaje-servicio fue tomando fuerza gracias a sus propósitos de servir y promover el bien social, pronto se encontró con diversos obstáculos para ser institucionalizado, ya que no había claridad sobre cómo generar aprendizajes disciplinares en los estudiantes ni mucho menos la forma de identificar las necesidades reales de las comunidades (Jacoby, 2015).

Además, fue blanco de críticas por no ser considerada una acción propia de la universidad, que tradicionalmente debía cumplir el rol de instruir a los estudiantes y generar conocimiento sin ocuparse del servicio público (Weaver y Kellogg, 2017).

No obstante, la reflexión que suscitó el informe Boyer (1990) dio paso a una mirada sobre la scholarship of engagement y el papel de las universidades en el ámbito público, en la construcción de la democracia y en las dinámicas sociales de los contextos (Weaver y Kellogg, 2017), lo que abrió la puerta a la institucionalización. De esta manera se logró superar la perspectiva pedagógica disciplinar que era dominante en la educación superior, consecuencia del boom ocasionado por el lanzamiento del Sputnik por parte de la Unión Soviética, lo que había consolidado un acuerdo en que el conocimiento era un arma estratégica contra el enemigo alejando la educación superior de sus fines formativos (García, 2019; Posner, 2005).

Recientemente, el engagement se convirtió en un camino para evidenciar y construir la relación entre lo que se enseña en las universidades y las necesidades sociales. En ese marco, la institucionalización se dio como un proceso gradual y requirió un trabajo en red sistemático (Dostillo y Perry, 2017). Goodman y Dean (1982) ya habían planteado que dicho proceso comienza por la toma de conciencia de una conducta o actividad en el ámbito individual, para luego pasar a un momento de mayor experimentación o puesta a prueba de dicha conducta o actividad, su posible replicabilidad, lo que da paso, luego de un relativo éxito y socialización, a una norma institucional y luego a la institucionalización. Los autores coinciden en que el logro de la institucionalización depende de la alineación con los objetivos institucionales (Furco y Holland, 2009), es decir, que el aprendizaje-servicio sea una estrategia que se pueda capitalizar para alcanzar las metas universitarias más amplias.

Por otra parte, existe un consenso en que los equipos que gestionan esta iniciativa al interior de las universidades deben tener un cuerpo de conocimientos específicos. Dostillo y Perry (2017) mencionan áreas como la pedagogía del compromiso comunitario, la investigación, los fundamentos de las teorías sobre la educación ciudadana y el desarrollo de estas competencias en los estudiantes, las orientaciones sobre el compromiso social en los académicos y la responsabilidad social universitaria, el desarrollo comunitario y organizacional, los mecanismos de gestión de alianzas con los socios comunitarios y el concepto de 
compromiso social. Como competencias clave de estos equipos aparecen también: un capital político al interior de la universidad, lo que le va a permitir al profesional relacionarse con distintas instancias dentro de la estructura organizacional; la habilidad de cultivar y gestionar adecuadamente las relaciones con los stakeholders; la capacidad para administrar el apoyo y los recursos institucionales esenciales; y la habilidad para apoyar y acompañar las partes interesadas (Weaver y Kellog, 2017). Adicionalmente, se incluyen conocimientos y competencias en marketing, planeación estratégica, liderazgo, resolución de conflictos, relaciones públicas y trabajo colaborativo, networking y pensamiento crítico, entre otros (Dostillo, 2017; Farmer-Hanson, 2017).

En Colombia, existen experiencias pedagógicas y curriculares con el componente de servicio, pero no se reconocen a sí mismas, la gran mayoría, dentro de esta categoría. Por ello, un reto importante, en universidades como la Javeriana, es hacer todo el proceso para que pase a ser una iniciativa institucional que recoja, por una parte, la amplia experiencia del trabajo que la Universidad tiene con comunidades, y por otra la riqueza de la experiencia consolidada internacionalmente sobre el aprendizaje servicio. Ello supone diversos retos, tanto para el equipo que está impulsando la iniciativa, como para los profesores que se unen, por lo que la sistematización agrega un valor especial para recoger las lecciones aprendidas y proyectar el trabajo posterior.

\section{Metodología}

El objetivo con el que se planteó la sistematización de experiencias de aprendizaje-servicio en la Javeriana fue el de recoger las lecciones aprendidas y las buenas prácticas desde las voces de quienes han participado en estos espacios para construir una ruta de institucionalización del aprendizajeservicio acorde con el contexto propio y natural Javeriano.

Se escogió como método la sistematización porque esta se comprende como un campo de producción de conocimiento colectivo que se da a partir de una mirada crítica de las experiencias, la cual se inscribe dentro de una perspectiva crítico social, permite reconstruir las practicas privilegiando los significados y sentidos que los actores le atribuyen a la experiencia (Torres, 1999), al tiempo que posibilita la construcción de aprendizajes y lecciones que contribuyen con el fortalecimiento del proceso analizado y otras experiencias similares (Jara, 2012).

El punto de partida que se tuvo para identificar los actores clave fue el trabajo documental que se desarrolló con base en la búsqueda de asignaturas en el catálogo de la Universidad. Estas se seleccionaron de acuerdo con los siguientes criterios: que tuvieran un componente teórico práctico y declararan de manera explícita en el sílabo la intención de servir a una comunidad y promover la formación integral de los estudiantes. En segundo lugar, se tuvieron en consideración las experiencias previamente conocidas por la Oficina de Responsabilidad Social, 
particularmente el trabajo realizado por los Programas Sociales de la Universidad Javeriana, en donde se desarrollan varias de las asignaturas de aprendizaje-servicio.

Posteriormente, se hicieron ocho entrevistas semiestructuradas por el carácter pragmático acerca de cómo los sujetos construyen y significan la experiencia (Tonon, 2009). En ellas se indagó acerca de las asignaturas, sus objetivos, las actividades de aprendizaje, la relación con los socios comunitarios y los significados de profesores y estudiantes entorno a esta experiencia. Adicionalmente, se realizaron tres grupos focales que contaron con la participación de 18 profesores y líderes de proyectos sociales. De acuerdo con Martínez (2015) la técnica de grupos focales permite centrar la discusión en temáticas particulares de interés para la investigación, en este caso, en las buenas prácticas y los retos asociados a las asignaturas de aprendizaje-servicio

Por otro lado, se realizaron dos entrevistas semiestructuradas para conocer casos exitosos de institucionalización del aprendizajeservicio en contextos con características similares al de la Universidad. Se abordó la experiencia de institucionalización de la Pontificia Universidad Católica de Chile y el caso de la Pontificia Universidad Javeriana, seccional de Cali.

También se hizo análisis de contenido, técnica que organiza la información de manera sistemática y rigurosa para la identificación de las categorías que aportan a la comprensión del problema
(Porta y Silva, 2003) y se contrastó a través de la triangulación. Se eligió esta técnica de análisis porque se buscó indagar información de distintas perspectivas y actores para enriquecer el proceso de investigación y darle fiabilidad.

\section{Resultados y discusión}

Esta aproximación al estado actual del aprendizaje-servicio en la universidad permitió identificar los significados que los distintos actores otorgan a estas iniciativas pedagógicas, las capacidades institucionales con las que se cuenta, las buenas prácticas y los retos que se evidencian en clave de institucionalización. A continuación, se presentan los principales hallazgos en cada categoría.

Con relación a las capacidades, que hace referencia a los elementos con los que cuenta la Universidad para la institucionalización, se identifica que a nivel misional y estratégico el proyecto educativo de la universidad está conectado de manera directa con el desarrollo de estas iniciativas, en tanto contempla que las funciones sustantivas deben estar al servicio de un país con regiones de características y necesidades diversas, entendiendo todo ello en el marco de la formación integral:

La formación integral de las personas que sobresalga por su calidad humana, ética, académica, profesional; y, la creación y el desarrollo de conocimiento y de cultura en una perspectiva crítica e innovadora, para el logro de una sociedad

Barbosa, A. y García, D. (2021). Aprendizaje-servicio en la Pontificia Universidad Javeriana seccional Bogotá, una experiencia de institucionalización en curso. RIDAS, Revista Iberoamericana de Aprendizaje Servicio, 12, 59-70. DOI10.1344/RIDAS2021.12.7 
justa, sostenible, incluyente, democrática, solidaria y respetuosa de la dignidad humana (Pontificia Universidad Javeriana, 2021, párr. 5).

En el proyecto educativo se evidencia que el currículo debe facilitar, tanto a los profesores como a los estudiantes, el reconocimiento de la realidad del país y su vinculación a procesos de transformación social. Estos enunciados están conectados de manera directa con la puesta en marcha e institucionalización del aprendizajeservicio, en la medida que permite materializar estas intenciones educativas en el currículo.

La misión, la planeación universitaria y las políticas institucionales también evidencian de manera concreta el compromiso de la Universidad con la transformación social del país y los aportes a la construcción de paz. Se destaca que la Universidad cuenta con una Política de Desarrollo Profesoral que contempla que:

Diversas modalidades de servicio se reconocen como parte de las actividades de los profesores de la planta de académicos: consultoría, educación continua y la proyección social que se desarrolla en el contexto de la Política de Responsabilidad Social de la Universidad. Las actividades de servicio son objeto de evaluación y los productos derivados de ellas hacen parte de la trayectoria académica del profesor; por lo tanto, son sujeto de valoración y reconocimiento (Pontificia Universidad Javeriana,

\author{
2017, p.7).
}

A nivel organizacional, se identifica que la Universidad cuenta con Proyectos Sociales Institucionales y unidades de proyección social gestionadas por diferentes facultades, así como consultorios (contable, jurídico, psicológico, entre otros) y una clínica jurídica con enfoque territorial. Todas las iniciativas tienen un enfoque de servicio orientado a favorecer las poblaciones más vulnerables de nuestro país. Estas iniciativas cuentan con equipos conformados por profesores y administrativos que conectan la sociedad con las actividades académicas. En estos centros se aglutinan gran parte de las actividades de las asignaturas, tanto de sus facultades, como de otras áreas, facilitando el trabajo interdisciplinario con enfoque territorial.

Por otro lado, se encuentra que los diferentes actores que participan en estas iniciativas significan la práctica social de manera muy positiva, destacando aspectos como su potencial para contribuir con la formación integral, los impactos positivos generados en los estudiantes, las comunidades y los profesores, así como el potencial de reflexión sobre el rol y que hacer de las carreras en la sociedad:

- "Venimos trabajando ya dos años y hemos podido conectar aquí practicantes $y$ algunos estudiantes de 1 y 2 semestre. Para los chicos más pequeños, para que entiendan que el diseño industrial está también llamado a aportar a la sociedad colombiana

Barbosa, A. y García, D. (2021). Aprendizaje-servicio en la Pontificia Universidad Javeriana seccional Bogotá, una experiencia de institucionalización en curso. RIDAS, Revista Iberoamericana de Aprendizaje Servicio, 12, 59-70. DOI10.1344/RIDAS2021.12.7 
más allá de lo que muchos que creen que es decorar, pintar, hacer sillas bonitas, sino cómo estamos aportando a la paz, a la equidad, a eliminar el narcotráfico, a generar actividades diferentes (Profesor Facultad de Arquitectura y Diseño Industrial, Pontificia Universidad Javeriana [PUJ], 2021).

En los significados que otorgan a estas asignaturas, destacan que ellas permiten al estudiante que conozcan cuáles son las realidades sociales, políticas y económicas de los diferentes contextos y cómo responder a ellas desde las disciplinas, ampliando el conocimiento de enfoques disciplinares, como es el caso de la carrera de Odontología en la que cuentan con dos asignaturas de aprendizaje servicio, a través de las cuales abordan el enfoque de los Determinantes Sociales en Salud y la Política de Salud Pública.

- "Digamos que para el proceso que nosotros manejamos se llaman prácticas comunitarias 1 y 2, la 1 se ve en tercero y la 2 en quinto semestre, lo que pretendemos es que el estudiante de Odontología empiece a reconocer un poco la realidad con que se va a encontrar, reconozca el contexto en el que está moviéndose, porque normalmente los odontólogos tenemos una facilidad, nos sentamos en un consultorio y nos aislamos del mundo y esperamos que el paciente nos llegue y solo vemos el contexto de ese lugar. Lo que buscamos con estas prácticas es que comprendan el contexto $y$ los determinantes sociales en salud" (Profesor Facultad de Odontología, PUJ, 2021).

Como buenas prácticas se evidencia la relación que existe en muchas de estas asignaturas con la espiritualidad, que cobra un sentido importante para los profesores por el potencial de trascendencia que permite en los estudiantes, entendiendo esta desde dos sentidos: por un lado, desde el servicio como una forma de salir de sí mismo y encontrarse en los otros; por otro lado, como espacios que se conectan y desarrollan desde el Paradigma Pedagógico Ignaciano, que es una forma de vivir la espiritualidad desde el encuentro con el otro, la acción y la reflexión.

Se evidencia en varios relatos que la acción de servir les permite salir se sí mismos y encontrarse con los otros, conocerlos, poder entender sus realidades $y$, desde ese conocimiento $y$ ayuda mutua, se genera un sentimiento de alegría, una transformación en sus diferentes dimensiones. Esto entra en diálogo con las intencionalidades de la educación jesuita en cuanto a la promoción de la fides (fe), que la entiende como el impulso al encuentro con los otros desde un amor desinteresado que sirve y obra a la justicia (Pontificia Universidad Javeriana Cali,2015).

- "He encontrado en esta asignatura la forma de servir, de que realmente lo que me ensañaron es verdad, que definitivamente uno se siente mucho mejor cuando puede 
ayudar a otras personas, pero me gusta más que mis estudiantes lo están descubriendo muy temprano, ya la gente se engancha y se siente feliz con su sitio, con su fundación, con el colegio" (Profesora Facultad de Ingeniería, 2021).

En otras asignaturas el proceso espiritual se da de una manera más intencionada, a través de la fundamentación del Paradigma Pedagógico Ignaciano que orienta el camino de relacionamiento, aprendizaje y acción que hace el estudiante con la comunidad: análisis de contexto, experiencia, reflexión, acción y evaluación.

- "En esta asignatura los estudiantes hacen un aprendizaje para a partir de esa comprensión y ese análisis del contexto, poder llevar acabo unas acciones de cara al servicio de las comunidades. Estas asignaturas se inspiran en el Paradigma Pedagógico Ignaciano. Acá se realiza un análisis de contexto, una evaluación, una reflexión y luego establecemos un accionar de intercambio con la comunidad a través de unos presaberes para lograr esa orientación y esa transformación en la comunidad" (Profesor Facultad Ingeniería, 2021).

La sistematización muestra que el impacto de estas asignaturas ha sido significativo tanto para los estudiantes como para las comunidades. Para los estudiantes, esta experiencia les permite aproximarse a realidades que para ellos a veces no son cercanas y así conocer las realidades de las personas. Lo anterior, los interpela sobre su rol en la sociedad y el servicio que pueden prestar para trasformar situaciones dolorosas:

- "Personalmente, siento que es esa cercanía o esa contextualización social con la realidad que se está viviendo, porque muchas veces vivimos en otra realidad donde no se ven las necesidades como tal de algunas poblaciones, y también ese aprendizaje de ¿Cómo puedo yo como ingeniero, desde mi área, contribuir para mejorarlo de alguna manera? También un aprendizaje que he tenido es que, en muchas ocasiones $y$ en contacto con la comunidad, ello nos comenta como...bueno. Por ejemplo, mi casa tiene...y nos cuentan algunos problemas que tiene la casa. Entonces nosotros como tratamos de, a partir de nuestra experiencia académica o lo que sabemos, como mira, te recomendamos hacer esto, pero ten cuidado con esto o con esa otra cosa" (Estudiante carrea Ingeniería Civil, PUJ 2021).

En el caso de las personas consultadas en la comunidad, el trabajo con los estudiantes y profesores les permite desarrollar procesos para el mejoramiento de sus comunidades y familias. Por ejemplo, en un escenario conversacional que se tuvo con líderes sociales participantes de un proyecto de legalización de previos que acompaña la Facultad de Ingeniería. Manifestaron

Barbosa, A. y García, D. (2021). Aprendizaje-servicio en la Pontificia Universidad Javeriana seccional Bogotá, una experiencia de institucionalización en curso. RIDAS, Revista Iberoamericana de Aprendizaje Servicio, 12, 59-70. DOI10.1344/RIDAS2021.12.7 
que para ellos era importante el apoyo que los estudiantes les brindaban para ayudar a comprender mejor los riesgos asociados al suelo del territorio y los factores ambientales, pero también las herramientas que podían construir a partir de estos conceptos técnicos para acceder a sus derechos y poder entablar un diálogo informado con el gobierno local.

Los retos que se evidencian en este proceso son, por una parte, que algunas de las asignaturas no cuentan con la continuidad ni el seguimiento de procesos con las comunidades, pues resultan más desde una iniciativa del profesor que desde una apuesta definida por parte de los programas académicos, y por otra, se encuentra que una porción de estas asignaturas es dirigida por profesores de cátedra, lo que puede afectar en algunos de los casos su continuidad.

El estudio también permitió identificar que existe desconocimiento al interior de la Universidad frente a las capacidades que la misma institución tiene y las iniciativas en curso. En el ejercicio de grupos focales se identificó que había profesores que no tenían conocimiento sobre el trabajo de otros colegas realizados en el mismo territorio o las capacidades de los proyectos sociales y las relaciones con las comunidades. En ese sentido, se configura como un reto importante los procesos de socialización de la metodología y la articulación de las iniciativas y capacidades existentes en la PUJ.

\section{Conclusiones}

El estudio permitió evidenciar cuales son las capacidades con las que cuenta la Universidad para la institucionalización del aprendizajeservicio, destacando principalmente su orientación social y trayectoria en iniciativas que aportan a la trasformación social. En este proceso, se destaca la motivación y compromiso por parte de actores clave como estudiantes, profesores y comunidades en las experiencias vividas y la recepción positiva por parte de ellos frente al proyecto de institucionalización. Por otro lado, también permitió identificar aspectos desafiantes como el desconocimiento de la metodología por parte de actores estratégicos, la falta de continuidad y seguimiento en algunos procesos, y la no identificación de las asignaturas bajo esta metodología que afecta su continuidad en los territorios.

Luego de contrastar las experiencias que se tomaron como casos de referencia para la institucionalización y las realidades la Universidad Javeriana se establece una posible ruta de institucionalización:

1. Fortalecimiento de una comunidad de práctica que motive y fomente las actividades de aprendizaje-servicio al interior de la Universidad y sea fuente de experiencia y conocimiento sobre el tema.

2. Visibilizar el aprendizaje-servicio y las prácticas exitosas que hoy existen en la Universidad. 
3. Simultáneo a lo anterior, fortalecer el relacionamiento y compromiso de las unidades académicas y administrativas directamente relacionadas con este tema, particularmente la Vicerrectoría Académica: Programas, Estudiantes, Desarrollo Profesoral y el Centro para el Aprendizaje, la Enseñanza y la Evaluación $(\mathrm{CAE}+\mathrm{E})$

4. Promover la articulación de las capacidades con las que hoy cuenta la Universidad en clave de aprendizaje-servicio. Es decir, motivar un mayor diálogo entre los programas sociales, apoyar el desarrollo de estrategias que permitan una mayor vinculación de asignaturas a estos espacios y acompañar el proceso de diseño e implementación de las estrategias de fomento $y$ transformación de cursos en aprendizaje-servicio.

5. Establecer la propuesta formativa y las herramientas pedagógicas para los profesores como un proceso continuo en la Universidad.

6. Concertar la marcación de las asignaturas de aprendizajeservicio en las carreas para garantizar la calidad de las mismas y la continuidad y seguimiento del impacto en los territorios.

\section{Referencias bibliográficas}

Boyer, E. (1990). Scholarship

Reconsidered: Priorities of the

Professoriate. The Carnegie Foundation for the advancement of teaching.

Dostillo, L. (2017). Planning a path forward: identifying the knowledge, skills, and dispositions of secondgeneration community engagement professionals. En L. Dostillo (Ed.) Community Engagement Professional in Higher Education (pp. 27-55). Campus Compact.

Dostillo, L., \& Perry, L. (2017). An explanation of community engagement professionals as professionals and leaders. En L. Dostillo (Ed.) Community Engagement Professional in Higher Education (pp. 1-26). Campus Compact.

Farmer-Hanson, A. (2017). Program administration and evaluation. En L. Dostillo (Ed.) Community Engagement Professional in Higher Education (pp. 79-97). Campus Compact.

Furco, A., \& Holland, B. (2009). Securing administrator support for service-learning institutionalization. En J. R. Strait y M. Lima (Eds.), The future of service-learning: New solutions for sustaining and improving practice (pp. 52-64). Stylus.

García, D. (2019). La formación de profesionales socialmente responsables en la universidad: una utopía posible en el currículo. Ediciones Uniandes.

Goodman, P., \& Dean, J. (1982).

Creating long term organization 
change. En Goodman (Ed), Change un organizations (pp. 226-279). JosseyBass

Jacoby, B. (2015). Service Learning Essentials: questions, answers, and lessons learned. Jossey-Bass

Jara, O. (2012). La sistematización de experiencias práctica y teoría para otros mundos posibles. Consejo de Educación de Adultos de América Latina (CEAAL).

Pontificia Universidad Javeriana Cali. (2015). Rasgos de nuestra identidad: características educativas e Intencionalidades Formativas Institucionales de la Pontificia Universidad Javeriana Cali. https://www2.javerianacali.edu.co/sites $\angle \mathrm{ujc} /$ files/node/fielddocuments/field document file/rasgos de nuestra identidad.pdf

Pontificia Universidad Javeriana. (2017). Política de Desarrollo del Cuerpo Profesoral. https://www2.javerianacali.edu.co/sites Lujc/files/politica de desarrollo del cu erpo profesoral.pdf

Pontificia Universidad Javeriana. (2021). Institucional. https://www.javeriana.edu.co/web/insti tucional

Porta, L., y Silva, M. (2003). El análisis de contenido de la investigación educativa. Anuario Digital de Investigación Educativa, (14), 388-406.

Posner, G. (2005). Análisis del currículo (3ra ed.). Mc Graw Hill.

Tonon, G. (Comp.). (2009). Reflexiones
Latinoamericanas sobre investigación cualitativa.

https://colombofrances.edu.co/wpcontent/uploads/2013/07/libro reflexio nes latinoamericanas sobre investigaci $\underline{n \text { cu.pdf }}$

Torres, A. (1999) La sistematización de experiencias educativas: reflexiones sobre una práctica reciente. Pedagogía y saberes, (13), 5-16.

https://doi.org/10.17227/01212494.13 pys5.15

Weaver, L., \& Kellogg, B. (2017). Attributes of Community Engagement Professionals Seeking to Institutionalize Community-Campus Engagement. En L. Dostillo (Ed.) Community Engagement Professional in Higher Education (pp. 118-138). Campus Compact.

Barbosa, A. y García, D. (2021). Aprendizaje-servicio en la Pontificia Universidad Javeriana seccional Bogotá, una experiencia de institucionalización en curso. RIDAS, Revista Iberoamericana de Aprendizaje Servicio, 12, 59-70. DOI10.1344/RIDAS2021.12.7 